\title{
The use of botulinum toxin in the treatment of adductor spasmodic dysphonia
}

\author{
R Whurr, M Lorch, H Fontana, G Brookes, A Lees, C D Marsden
}

\begin{abstract}
Botulinum toxin injections have been used to treat 31 patients with adductor spasmodic dysphonia. Injections of $3 \cdot 00-3 \cdot 75$ units of botulinum toxin were performed bilaterally into the thyroarytenoid muscle. This treatment significantly decreased the standard deviation of the fundamental frequency of the speech sample, indicating a reduction in the variability of pitch amongst patients. A total of $96 \%$ of patients' subjective diary reports showed an improvement with a median of 7 days to peak effect and a 5 week duration of peak effect.
\end{abstract}

(F Neurol Neurosurg Psychiatry 1993;56:526-530)

Adductor spasmodic dysphonia (SD) is a chronic voice disorder of adult onset and unknown aetiology. SD is not a "spastic" disease, but is a focal laryngeal dystonia which should be differentiated from vocal tremor and myoclonus. ${ }^{1-3}$ Although it is often erroneously labelled "spastic" dysphonia, no signs of spasticity have been found in the vocal folds of patients with spasmodic dysphonia. ${ }^{2}$ This voice disorder is characterised by regular frequent breaks in phonation, staccato-like catches, pitch breaks and variations in pitch. Attempts to speak are usually accompanied by effortful, jerky, groaning, and strained phonation.

Spasmodic dysphonia was first described by Traube, ${ }^{4}$ who suggested that SD was an hysterical illness. A psychiatric aetiology was supported by Beck, ${ }^{5}$ Berendes, ${ }^{6}$ and $\mathrm{Kiml},{ }^{7}$ and this concept led to a variety of treatments, including psychotherapy, acupuncture, hypnosis, biofeedback, faradism and drug therapy, all of which had little benefit. A physical cause of SD was first proposed in 1874 by Schnitzler ${ }^{8}$ who described two patients with "cramping of the vocal cords and forced voice". He called this entity "spastic aphonia". In 1939, Critchley" described two patients with a vocal disorder who had associated neurological signs and concluded that the site of the pathological process was likely to be the cerebellum or the basal ganglia.

More recent studies have linked SD with dystonia. The association of SD with Meige's syndrome led to the disorder being redefined as a focal form of dystonia, one of a group of movement disorders which are characterised by prolonged muscle contractions with twist- ing spasms, which may have additional myoclonic or tremulous components. ${ }^{11011}$

Fibreoptic observations in patients with SD have shown rapid twitch-like movements and forceful spasm-like jerking of the vocal folds, and oscillations of the arytenoids. Forceful, rapid, jerking spasms are noted in the velum as well as in the superior pharynx and tongue. Such abnormal movements of the vocal folds may even be evident during quite respiration. These are exacerbated during phonation. ${ }^{12}$

The growing acceptance of the neurological aetiology of SD led to a change in approach to the treatment of the disorder. Dedo $^{13}$ practised surgical resection of the recurrent laryngeal nerve. It was thought that unilateral vocal fold paralysis would result in compensation due to the strong adductive forces in the unparalysed contralateral side. Laryngeal nerve resection provided initial relief, but relapses were common. Long term results were disappointing, with reports in some cases of symptoms being made worse. ${ }^{14}$ The cause for the relapse was thought to be due to the hyperadduction of the non-denervated cord with exaggeration of the dystonic spasms, or reinnervation on the surgical side. ${ }^{16}$ Other forms of surgery have included arytenoid replacement, laser therapy, spinal cord stimulation, and even thalamotomy, all with no benefit or transient effects. Pharmacological intervention has included the use of anticholinergics, benzodiazepines, baclofen and carbamazepine. ${ }^{16}$ Only mild benefit from clonazepam and anticholinergics has been reported.

Recently, injections of botulinum toxin into targeted muscular sites have proved to be beneficial in the treatment of certain focal dystonias. ${ }^{1719}$ Botulinum toxin acts presynaptically to block the calcium dependent release of acetylcholine. When injected in low doses the toxin causes local weakness in the muscle into which it is injected. This eliminates the abnormal muscle contractions while preserving functional ability. The effect of the toxin is temporary due to the re-sprouting of axons which form new neuro-muscular junctions.

The use of botulinum toxin treatment in spasmodic dysphonia of the adductor type is currently being tested in several centres. ${ }^{3161920-24}$ Table 1 summarises the various methods of botulinum toxin treatment currently being pursued. The potency of toxin being used in the UK (Dysport) differs from that being used in the USA (Oculinum) 
Table 1 Summary of studies of the use of botulinum toxin in the treatment of SD

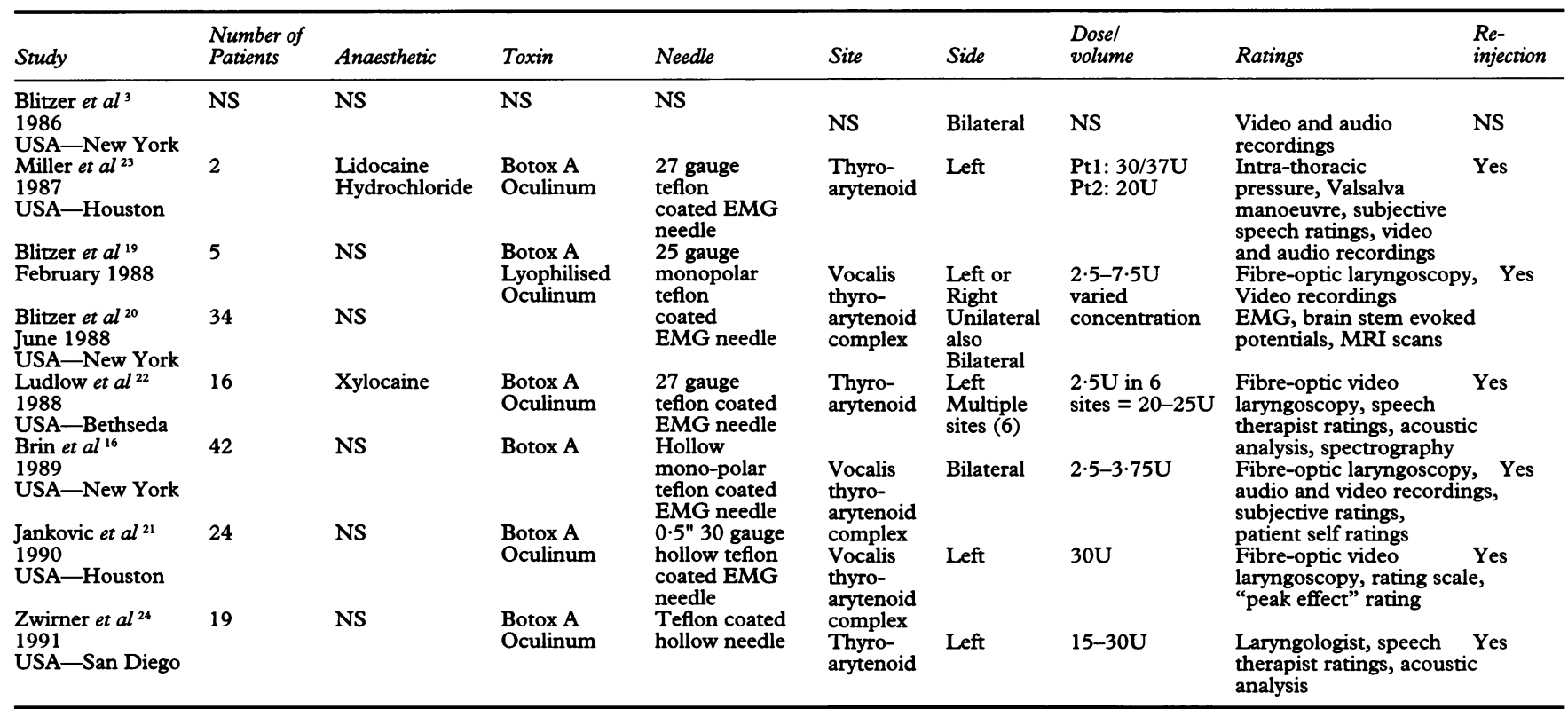

There are differences in the techniques reported for dose, site, and number of injections of botulinum toxin. In spite of these differences, all centres utilising botulinum toxin in the treatment of adductor SD claimed good results for improving the voice. Symptoms generally return after the restoration of vocal cord movement over a period of about 12 weeks. There have been no reports of patients becoming refractory to therapy. Brin et $a l^{16}$ found that there was a trend for a progressively longer period of benefit between injections.

The methods of evaluation of the results of botulinum toxin injections on vocal quality also have varied. Methods of assessment have included neurological, speech therapy/pathology, fibre-optic laryngoscopy, EMG, audio and video recording. Evaluation of results has commonly included subjective speech/video recordings, though Ludlow ${ }^{22}$ and Zwirner ${ }^{24}$ included objective acoustic analysis of speech. Other methods have been patient diaries ${ }^{26}$ and measures of interthoracic pressure and Valsalva manoeuvres. ${ }^{23}$

This paper describes the procedure used to assess, treat and evaluate treatment of patients with spasmodic dysphonia using botulinum toxin.

\section{Method}

Subjects

Between December 1988 and April 1991, 49 patients with suspected spasmodic dysphonia of the adductor type were referred to the spasmodic dysphonia (SD) clinic. Eighteen patients were removed from the trial: 12 were found on assessment not to have SD, 3 patients were treated elsewhere ( 1 found the travel too far, 1 was too unwell and 1 patient died). Thirty one patients entered the trial, 11 male, 20 female with a mean age at the time of initial assessment of 57 years (range
31-82). The mean age of the male subjects was 46 years (range 31-65); that of females was 63 years (range 43-82). Mean duration of symptoms was 5 years 2 months (range 11 months-18 years). There was no difference in mean duration of symptoms for males and females.

Fourteen of the 31 subjects (45\%) had associated neurological signs: 10 female $(50 \%), 4$ male (36\%). These signs were: a) tremor ( $n=5$, female patients) of various types-2 had hand tremor, 2 had head tremor (including voice tremor), 1 head, voice and hand tremor; $b$ ) dystonias ( $n=8,3$ male and 5 female) of various types-2 torticollis, 1 multifocal dystonia, 1 torticollis and writers cramp, 2 blepharospasm, 2 Cranial dystonia; c) Parkinsonism ( $\mathrm{n}=1$, male) (see table 2).

Twenty two patients $(71 \%)$ had had previous treatments including: recurrent laryngeal nerve crush, drugs, faradism, hypnotherapy/ hypnosis, acupuncture, reflexology, chiropractice, herbalism, psychotherapy, speech therapy. All found the treatments to have had no lasting effect on the voice. Four patients were receiving drug treatment at the time of their initial injection of botulinum toxin, they reported that the drugs were having no effect on their voice.

No control subjects were studied, because of the remarkable efficacy of this treatment, shown not only by this study but also by other studies (see table 1). A placebo controlled trial would probably not be ethically justified.

\section{Assessment Procedures}

Each patient had initial assessment by a team of a neurologist, a neuro-otolaryngologist and a speech-language therapist. The laryngological assessment was performed using either a flexible fibreoptic or rigid endoscope connected to a video camera. The speech-language therapist assessed each patient on a voice pro- 
Table 2 Patient characteristics

\begin{tabular}{|c|c|c|c|}
\hline Sex & Age range & $\begin{array}{l}\text { Duration of } \\
\text { voice symptoms } \\
\text { (range) }\end{array}$ & $\begin{array}{l}\text { Associated } \\
\text { neurological signs }\end{array}$ \\
\hline $\begin{array}{l}\text { Male } \\
n=11\end{array}$ & $31-65$ years & $1-18$ years & $\begin{array}{l}\mathrm{n}=4 \\
1 \text { cranial dystonia, } \\
1 \text { torticollis, } 1 \text { torticollis and } \\
\text { writers cramp, } 1 \text { Parkinsonism. }\end{array}$ \\
\hline $\begin{array}{l}\text { Female } \\
\mathrm{n}=20\end{array}$ & $43-82$ years & 11 months- 15 years & $\begin{array}{l}\mathrm{n}=10 \\
2 \text { head and voice tremor, } 2 \text { hand } \\
\text { tremor, } 1 \text { head hand and voice } \\
\text { tremor, } 2 \text { blepharospasm, } \\
1 \text { torticollis, } 1 \text { multifocal } \\
\text { dystonia, } 1 \text { cranial dystonia. }\end{array}$ \\
\hline
\end{tabular}

tocol which was audio and video recorded. This vocal protocol included the reading of a passage (The North Wind and the Sun) which would be used for later analysis. The audio recording was made using a UHER CR 160 AV stereo cassette recorder in conjunction with a UHER M534/A cardiod directional microphone. Filters were used to extract extraneous ambient background noise. All recordings were carried out in a video recording studio. Each patient was reassessed by the team at each follow up visit.

\section{Botulinum toxin treatment procedure}

The toxin Clostridium Botulinum toxin Type A (Dysport) is obtained from Porton Products Ltd as a freeze dried toxin-haemagglutonin preparation. Fifty nanograms is equivalent to 2000 mouse-units. To begin with we diluted in normal saline to a concentration of 25 units per $\mathrm{ml}$. Now we use a concentration of 40 units per $\mathrm{ml}$.

The injection procedure used followed that of Brin et al. ${ }^{16} \mathrm{~A}$ small dose of botulinum toxin was injected into both thyroarytenoid muscles (that is, left and right). Before the injection $2 \%$ lignocaine was injected down through the cricothyroid membrane to provide local anaesthesia. EMG recordings were employed to localise the thyroarytenoid muscle. A 26 gauge hollow monopolar teflon coated needle was used with a skin reference (clavicle) and a ground in position. The needle was advanced through the cricothyroid membrane in a superior and lateral direction until a crisp motor unit interference pattern was heard. When the monopolar teflon coated laryngeal EMG needle was in the correct position, the toxin was administered through the same needle which was then withdrawn.

The original injection dose was 3.75 units in $0.15 \mathrm{mls}$. Recently the dose was altered slightly to 3 units in $0.075 \mathrm{mls}$. The first 22 patients in the series received the original larger dose; 9 subsequent patients received the new smaller dose.

The procedure was tolerated well by the majority of patients, although at times coughing and gagging occurred. The use of Merocaine lozenges (benzocaine cetylopyridium chloride) sucked 30 minutes before the injection has proved useful to suppress the tendency to cough during the injection.

Follow up

After the injections the patients were requested to keep voice diaries recording the occur- rence, quality, and duration of any negative side effects and of positive improvement in their voices after treatment. Speech samples were collected when patients returned to the SD clinic for follow up.

Patients were usually seen for a follow up assessment 2-3 months after their injection and, if appropriate, they were re-injected with the same dose bilaterally. Patients were again assessed by the speech-language therapist who recorded the vocal protocol. The interval between the recordings was not the same for every subject (mean interval $=10$ weeks, range 2-30 weeks). As the SD clinic draws patients from great distances, many could only attend the clinic for follow up visits when their voices were beginning to deteriorate. Typically patients were found to require re-injection at this time. The post-injection recordings were most commonly made at the point when the voice was beginning to deteriorate and had passed the peak period of maximum benefit.

\section{Speech analysis}

The effect of botulinum toxin injections on the patients' vocal quality was investigated with two different measures: 1) objective acoustic speech analysis and 2) patients' subjective diary reports.

\section{Acoustic analysis}

The audio taped recordings of the patients' reading of The North Wind and the Sun were analysed using the Visispeech speech analysis package (RNID) on a BBC Master computer. The Visispeech speech analysis programme measures the fundamental frequency of the voice over the duration of the entire speech sample. The data are scaled into 85 "bins" or compartments, and measures of central tendency, variance and standard deviation are calculated.

The measures of standard deviation of the distribution of frequency in the entire speech samples were compared pre- and post-injection. This measure can be taken as an indication of regularity of pitch of voice. Thus a voice with many pitch breaks and variations in pitch has a high standard deviation value. The standard deviation values pre-injection and post-injection were compared using a Wilcoxon Signed-Ranked Test.

\section{Patient diaries}

The analysis of the patients' diary records was carried out to determine: 1) the length of delay before the benefits of the injections were noted; 2) the duration of peak effect, that is, the length of time the voice was at its maximum improvement before any deterioration was noted, and 3 ) the duration of response, the length of time the patient received any benefit from the injection.

\section{Results}

Acoustic analysis

Of the 31 patients' audio recordings both preand post-injection only 22 were technically 
Figure Values for standard deviation of fundamental frequency pre and post botulinum toxin injection for adductor spasmodic dysphonia.

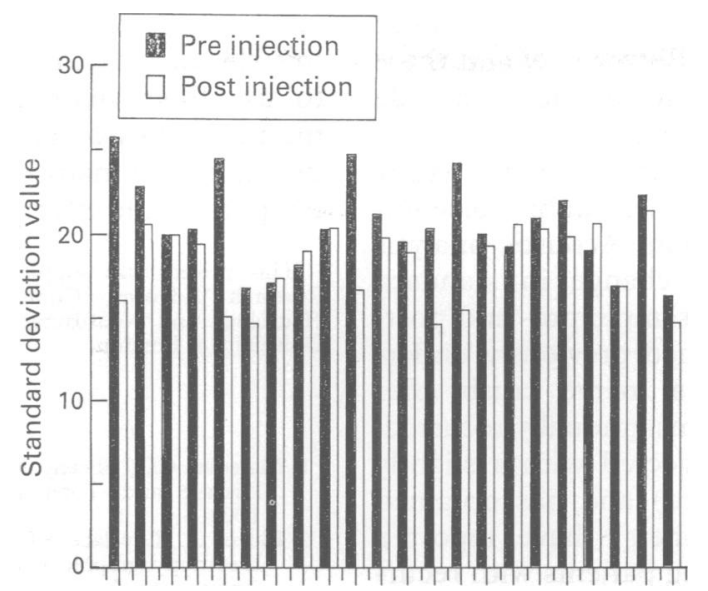

acceptable for acoustic analysis. These 22 speech samples were analysed for the standard deviation of long term fundamental frequency. The measures of standard deviation of the speech samples recorded post-injection were found to be significantly lower than the pre-injection values $(p<0.005)$ using the Wilcoxon signed ranks test (see figure).

\section{Beneficial effects}

At the follow up visit the vast majority of patients $(96 \%)$ in our study reported improvement in their voice, some reporting it "back to normal", and many gave very favourable reactions speaking of their increased confidence at work and socially since the change in their voices. Only a proportion of the 31 patients successfully kept voice diaries. The data available was analysed on diaries from 24 patients. The median time for onset of effects post-injection was 7 days ( $n=23$, range $1-18$ days). The median duration of peak effects was 5 weeks $(n=24$, range 1-12 weeks). The vast majority of our patients, at the time of their second injection, reported that their voice quality, though deteriorated from a peak level, was still an improvement on their pre-injection voice quality.

Beneficial vocal effects after injection reported by the patients included: reduced number of pitch and voice breaks, increased volume and increased overall ease of production (that is, reduced effortfulness).

\section{Adverse effects}

Temporary side effects were reported by 8 out of 22 patients $(25 \%)$. These negative side effects included: some dysphagia for fluids (without aspiration) ( $n=5)$, weak cough $(n=2)$ and slight pain at the site of injection $(n=2)$.

\section{Discussion}

This study shows that bilateral botulinum toxin injections into the thyroarytenoid provide an effective treatment for spasmodic dysphonia of the adductor type. This further confirms evidence of previous studies that have indicated the beneficial effects of botulinum toxin on this voice disorder. ${ }^{316} 19-2426$
At the time we started this study, the largest experience of botulinum toxin injections for adductor spasmodic dysphonia had used bilateral injections. ${ }^{16}$ Others, however, have employed unilateral injections. The relative merits of these two approaches have not been assessed formally. In theory, a unilateral injection might be safer, but bilateral injections might produce the same physiological result with smaller doses.

The analysis of standard deviation of fundamental frequency of a speech sample provided a useful objective assessment of the effects of treatment on the regularity of pitch, an important indicator of vocal quality. The finding of decreased standard deviation of frequency distributions after injection indicated an increase in regularity of the voice and a decrease in pitch breaks and variations in pitch. This objective acoustic measure confirmed the subjective assessment of the speech-language therapist and the patients' own reports. These findings on long term frequency measures substantiate those reported by Zwirner et al ${ }^{24}$ comparing the standard deviation of fundamental frequency for a sustained vowel segment pre- and post-injection with botulinum toxin. They also found that standard deviation values were lower after injection.

As the post-injection assessments and recordings were not necessarily obtained during the peak period of maximum benefit, but at a time when the positive effects of the treatment were presumably already on the wane, this analysis can be taken conservatively. This evidence suggests that even when the peak period is over there is still a marked and lasting improvement in the voice compared with the voice pre-injection. This supports the findings reported by Brin et al ${ }^{16}$ that vocal symptoms do not recur after injection to the same level of strength and intensity as before botulinum toxin treatment.

Patients reports of vocal effects after injection indicated that onset of beneficial effects occurred at day 7 on average. The duration of peak voice benefit was typically 5 weeks. Only $25 \%$ of patients reported any negative side effects which were transitory in nature. Jankovic and Brin ${ }^{25}$ state that a higher incidence of side effects have been reported when using the British toxin, Dysport, compared with the American toxin, Oculinum. They suggest that this might be accounted for by the higher potency of Dysport compared with Oculinum (1 nanogram of Dysport contains 40 units whereas 1 nanogram of Oculinum contains 2.5 units, where a "unit" is a standard measure of potency of commercially available toxin). However, when the incidence of side effects in the study by Blitzer ${ }^{26}$ which utilised low dose bilateral injections (range of doses 1.25-3.75 units) was compared with the results obtained during this study it was seen that the incidence was very similar. Blitzer and Brin $^{26}$ reported $45 \%$ of patients with a short period of breathy dysphonia and $22 \%$ with mild dysphagia for fluids. For low dose bilateral injections for 
adductor SD the results reported here concur with those obtained by Blitzer et al and there is no evidence for a higher incidence of side effects using the British toxin.

Only one of the 31 patients in the study failed to gain an improvement in his voice following the initial injection. Acoustic analysis revealed very minimal change in standard deviation of the speech sample pre- and postinjection. This patient had previously undergone recurrent laryngeal nerve crush after which the voice symptoms gradually recurred. He has since been reinjected with a slightly larger dose (5 units/cord) and has obtained an excellent result. Others ${ }^{16}$ have reported positive results in treating patients with recurrent nerve crushes.

Three patients had voice tremor in addition to SD. The injections of botulinum toxin alleviated the symptoms of SD but the voice tremor remained. Though the tremor did not markedly impede the speaker's intelligibility it was perceived by them as socially disabling.

In the present report, only data pertaining to the effects of the first injection have been analysed. All patients have, however, received follow up injections, and data from these suggests that the injection continues to have a beneficial effect. All 31 patients have continued with injections every 2-4 months, as appropriate, for periods of up to 3 years, with continued benefit.

The majority of treated patients derive the most dramatic improvement after the first injection of botulinum toxin. Most patients require subsequent multiple injections at varying intervals to maintain spasm-free vocal performance. Graded responses of muscle weakness can be obtained by using low doses of botulinum toxin. Once the patient's sensitivity to treatment is determined, an individualised protocol can be used for future injections, with small dose "top-ups" injected into one or both vocal cords. Low dose bilateral injections into the thyroarytenoid is suggested as the preferred method of treatment for SD of the adductor type.

Occasionally, patients report that they did not have any (positive or negative) effects following an injection, when previously (and subsequently) they have had a good response. At the outset it appeared that the botulinum toxin had not taken effect in these cases. However, these rare instances of failure of the injection to produce an effect may be linked to difficulties in the injection procedure itself, particularly if there have been problems obtaining a satisfactory EMG signal. Our experience therefore indicates the importance of using EMG to locate the thyroarytenoid muscle. Not every "failed" injection can probably be attributed to this factor, and further investigation of this problem is needed.

In conclusion, the use of botulinum toxin in SD is an efficient method of treatment for this chronic voice disorder. It enables patients to use their voice effortlessly and to communicate easily -in some instances for the first time for many years, with very few and temporary side effects.

This project was supported by a grant from the Joint Research Advisory Committee, National Hospital for Neurology and Neurosurgery, and Institute of Neurology, University of London.

1 Marsden CD, Sheehy MP. Spastic Dysphonia, Meige's Disease and Torsion Dystonia. Neurology 1982;32 1202-3.

2 Blitzer A, Lovelace RE, Brin MF, Fahn S, Fink ME. Electromyographic findings in focal laryngeal dystonia (spastic dysphonia). Ann Otol Rhinol Laryngol 1985; 94:591-4.

3 Blitzer A, Brin MF, Fahn S, Lovelace RE. Botulinum toxin for the treatment of spastic dysphonia. Laryngoscope 1986;96:1300-1.

4 Traube L. Spastiche form der nervosen heiserkeit. In: Traube L, ed. Gessammelte Beitraege zur Pathologie und Physiologie, vol 2. Berlin: Hirschwald, 1871: 674-8.

5 Beck $K$. Uber Erfahrungen mit Stimmstoerungen bei Kriegsteilnehmen. Passow-Schaefer Beitrage. Anat Ohres Usw 1918;11:130.

6 Berendes J. Zur Ensteheng und Behandlung der Dysphonia Spatica. Z Hals usw Heilk 1938;44:78-84.

$7 \mathrm{Kiml}$ FJ. Recherches experimentales de la dysphonia spastique. Phoniatric (Basel) 1965;17:241-301.

8 Schnitzler J. Klinischer atlas der laryngologic nebst anleitung zur diagnose und therapie der krankheiten des kehlolopfes und der luftrohre. Wein: Braunmuller, 1885.

9 Critchley M. Spastic dysphonia (inspiratory speech). Brain 1939;62:95-103.

10 Golper L, Nutt JG, Rau MT, Coleman RO. Focal cranial dystonia. $\mathcal{F}$ Speech Hearing Disorders 1983;48:128-34.

11 Jacome DE, Yanez GF. Spastic dysphonia and Meige's disease. Neurology 1980;30:349.

12 McCall GN, Skolnick ML, Brewer DW. A preliminary report of some atypical movement patterns in the tongue, palate, hypopharynx, and larynx of patients with spasmodic dysphonia. $\mathcal{f}$ Speech Hearing Disorders 1971;36:446-70.

13 Dedo HH. Recurrent laryngeal nerve section for spastic dysphonia. Ann Otol Rhinol Laryngol 1976;85:451-9.

14 Aronson A, DeSanto L. Adductor spastic dysphonia: 3 years after recurrent nerve resection. Laryngoscope 1983;93:1-8.

15 Fritzell B, Feuer E, Knutsson E, Shiratzki H. Experiences with recurrent laryngeal nerve resection for spastic dyswith recurrent laryngeal nerve resection for spastic

16 Brin MF, Blitzer A, Fahn S, Gould W, Lovelace RE. Adductor laryngeal dystonia (spastic dysphonia): Treatment with local injections of botulinum toxin (Botox). Movement Disorders 1989;4:287-96.

17 Elston JS. The clinical use of botulinum toxin. Seminars in Ophthalmology 1988;3:249-60.

18 Marsden CD, Quinn NP. The dystonias. BMf 1990;300:139-44.

19 Blitzer A, Brin MF, Fahn S, Lovelace RE. Localized injections of botulinum toxin for the treatment of focal laryngeal dystonia (spastic dysphonia). Laryngoscope 1988(a);98:193-7.

20 Blitzer A, Brin MF, Fahn S, Lovelace RE. Clinical and laboratory characteristics of laryngeal dystonia: a study laboratory characteristics of laryngeal dystonia

21 Jankovic J, Schwart K, Donovan DT. Botulinum toxin treatment of cranial-cervical dystonia, spasmodic dysphonia, other focal dystonias and hemifaciat spasm. phonia, other focal dystonias and hemifacin

22 Ludlow CL, Naunton RF, Sedory SE, Schulz GM; Hallet $M$. Effects of botulinum toxin injection on speech in adductor spasmodic dysphonia. Neurology 1988a;38: 1220-5

23 Miller RH, Woodson GE, Jankovic J. Botulinum toxin injection of the vocal fold for spasmodic dysphonia. Arch Otolaryngol Head Neck Surg 1987;113:603-5.

24 Zwirner P, Murry T, Swenson M, Woodson GE. Acoustic changes in spasmodic dysphonia after botulinum toxin injection. $\mathcal{F}$ of Voice 1991;5,1:78-84.

25 Jandovic J, Brin M. Therapeutic uses of botulinum toxin. New Engl f Med 1991;324:1186-94.

26 Blitzer A, Brin M. Laryngeal dystonia: a series with botulinum toxin therapy. Ann Otol Rhinol Laryngol 1991; 100:85-9. 